\title{
Evaluation of the Osteogenic Potential of Growth Factor-Rich Demineralized Bone Matrix In Vivo
}

\author{
Shan-Huey Yu, ${ }^{*}$ Hsun-Liang Chan,${ }^{\dagger}$ Li-Yen Chong, ${ }^{\dagger}$ Yi-Han Jheng, ${ }^{\S}$ and Po-Chun Chang*§
}

Background: The study evaluates the osteogenic properties and biocompatibility of growth factor-rich demineralized bone matrix (GDBM) by comparing with cancellous mineralized bone matrix (CMBM) and anorganic bovine bone matrix (ABBM).

Methods: Thirty-six Sprague-Dawley rats were used $(\mathrm{n}=$ 6/group/time point). To assess biocompatibility and osteoinductivity, the respective bone matrices were randomly placed in subcutaneous pouches for 7 and 28 days and evaluated by histology and osteopontin expression. Osteoconductivity was assessed by randomly implanting respective bone matrices in osteotomies on femurs for 14 and 28 days and evaluated by microcomputed tomography and histology.

Results: Neither acute inflammation nor mineralized tissue was noted in any of the subcutaneous specimens, whereas expression of osteopontin was more prominent in the GDBM group. Among the femoral specimens, the greatest relative bone volume (bone volume [BV] divided by trabecular volume [TV]) and trabecular thickness was noted in the ABBM group at both time points, whereas less BV/TV was noted in GDBM group at day 14. Residual matrix particles were noted in all examined groups at both time points, without significant differences regarding defect fill between groups. The GDBM group presented similar levels of newly formed bone compartment and marrow space to those of the ABBM group.

Conclusions: GDBM demonstrated acceptable biocompatibility and osteogenic potential comparable to ABBM in vivo. Further investigations in a more clinically relevant model are warranted. J Periodontol 2015;86:36-43.

\section{KEY WORDS}

Biocompatible materials; bone matrix; bone regeneration; cytokines; periodontics; tissue scaffolds.

* Department of Dentistry, National Taiwan University Hospital, Taipei, Taiwan.

$\dagger$ Department of Periodontics and Oral Medicine, University of Michigan, Ann Arbor, MI.

$\neq$ Faculty of Dentistry, National University of Singapore, Singapore.

$\S$ Graduate Institute of Clinical Dentistry, School of Dentistry, National Taiwan University, Taipei, Taiwan.
$\mathrm{B}$ one grafts have been widely used to aid in the reconstruction of osseous dentoalveolar defects as well as atrophic alveolar ridge augmentation before implant placement. ${ }^{1,2}$ These range from autogenous bone grafts (ABGs) and allografts to xenografts and alloplast or synthetic graft materials. ${ }^{3}$ Ideally, the grafting material must present adequate osteogenic, osteoinductive, or osteoconductive properties to achieve bone regeneration. ${ }^{4}$ ABG is considered the gold standard grafting material because it possesses the three abovementioned properties, and the use of autografts diminishes the risk of infectious disease transmission. ${ }^{5}$ Despite ABG being viewed as a reliable treatment option, it always carries an increased risk of donor-site morbidity and limited availability. Freeze-dried bone allograft (FDBA) appeared to be an attractive alternative. ${ }^{6}$ However, FDBA only served as a scaffold and did not demonstrate a result comparable to ABG. ${ }^{7}$

Osteoinductive factors (i.e., bone morphogenetic proteins [BMPs]) insulated by the mineral compartment could be released only after demineralizing the bone matrix (i.e., demineralized FDBA [DFDBA]). Hence, DFDBA was considered to lead to a better osteogenic potential relative to FDBA. $^{8}$ However, the osteoinductivity of DFDBAs could vary according to the processing protocols, carriers, and the source of allograft. ${ }^{9}$ In 
addition, the advent of efficient, safe processing and sterilization techniques of ABG and FDBA have spawned research into alternative substitutes, such as xenografts with unlimited supply and no additional donor site required for periodontal regenerative procedures. ${ }^{10}$

Advances in molecular technology have demonstrated that growth factors are a potential biologic agent to enhance craniofacial reconstruction, periodontal regeneration, and implant site preparation. ${ }^{11}$ Growth factors are naturally occurring polypeptides that target the appropriate cells to carry out a specific biologic function. The most commonly reviewed growth factors in the field of dentoalveolar regeneration are platelet-derived growth factor (PDGF) and BMPs. ${ }^{12,13}$ By promoting the recruitment of mesenchymal stem cells and differentiating to osteoprogenitor cells, BMPs have been identified as the most potent osteoinductive elements and are now of considerable interest as therapeutic agents for reconstructing dentoalveolar defects as well as facilitating osseointegration of dental implants. ${ }^{14}$ On the other hand, PDGF is a chemotactic and mitogenic agent that has demonstrated a beneficial effect in the regeneration of the human periodontium. ${ }^{13,15}$ An in vitro study demonstrated that recombinant human BMP-7 (rhBMP-7) combined with DFDBA stimulated the early osteoblastic differentiation of human periodontal ligament cells and suggested that DFDBA could serve as a carrier for the growth factors. ${ }^{16}$ Being a controlled-release carrier, the combination of DFDBA and growth factors provided good clinical results in accelerating the healing of the periodontal defects. ${ }^{17,18}$ Recently, a BMP-containing growth factor-rich DFDBAll was introduced as a clinical application for socket preservation and ridge augmentation. However, little is known of its osteoinductivity and osteoconductivity relative to other bone grafting materials.

This study aims to compare the properties of growth factor-rich demineralized bone matrix (GDBM) with other clinically available bone grafting materials, including cancellous mineralized bone matrix (CMBM) and anorganic bovine bone matrix (ABBM), based on evidence from microcomputed tomography (micro-CT) imaging and histology in vivo.

\section{MATERIALS AND METHODS}

\section{Animal Models}

All animal procedures were performed under protocol no. 20130054 approved by the Institutional Animal Care and Use Committee of the National Taiwan University. Based on the results from the authors' previous study using the same species of animals with a similar size of osteotomy, ${ }^{19}$ at least $20 \%$ difference was assumed in the relative bone volume (bone volume [BV] divided by trabecular volume [TV]) between the control and GDBM groups, $80 \%$ power, $\alpha=0.05$, and normal distribution and equivalent variance of the samples. As a consequence, a total of 36 ( $\mathrm{n}=6 /$ group/time point) 8week-old male Sprague-Dawley rats were used in this study.

The biocompatibility and osteoinductivity of the graft materials were evaluated in the first part of the study (Fig. 1A). Bilateral subcutaneous pouches were created on the dorsum of the rats under general anesthesia with intraperitoneal injection of $90 \mathrm{mg} / \mathrm{kg}$ ketamine and $10 \mathrm{mg} / \mathrm{kg}$ xylazine described in a previous study. ${ }^{20}$ Each pouch was randomly filled with $\approx 300 \mu \mathrm{L}$ of one of the following grafting materials: 1) GDBM;" 2) CMBM;\# and 3) ABBM.** The incision was then closed by surgical staples. All animals were given ampicillin (263 mg/L) in drinking water for 5 days and euthanized by $100 \%$ carbon dioxide at day 7 or 28.

The osteoconductivity of the graft materials was evaluated in the second part of the study (Fig. 1B). Under general anesthesia as described in the first part, a 2.6-mm-diameter and 1.2-mm-depth osseous defect was created bilaterally on the middiaphysis of the femur by a customized drill with copious normal saline irrigation. Each defect was randomly filled with one of the graft materials (GDBM, CMBM, or ABBM) or left empty (control). All animals were given ampicillin for 5 days and euthanized by $100 \%$ carbon dioxide at day 14 or 28 .

Upon sacrifice, the tissue of the investigated areas (subcutaneous pouches and femurs) was harvested and stored in $10 \%$ formaldehyde at $4^{\circ} \mathrm{C}$ for subsequent examinations.

\section{Micro-CT Assessments}

An x-ray CT scanner ${ }^{\dagger \dagger}$ was used to examine the harvested femurs. The images were reconstructed using the Shepp-Logan algorithm to achieve an effective pixel size of $19 \mu \mathrm{m}$, and the threshold of the image was determined by a local edge detection algorithm, ${ }^{21}$ which showed the strongest correlation to the histomorphometry among the current thresholding algorithms from the authors' previous study. ${ }^{22}$ The entire defect area was selected as the region of interest (ROI), and the micro-CT parameters, including BV/TV, trabecular thickness (Tb.Th), trabecular separation (Tb.Sp), and trabecular number

\footnotetext{
Accell, Keystone Dental, Burlington, MA.

I Keystone Dental.

\# Puros, Zimmer Dental, Carlsbad, CA.

* Bio-Oss, Geistlich Biomaterials, Wolhusen, Switzerland.

$\dagger \dagger$ Shimadzu SMX-100CT x-ray CT scanner, Shimadzu Corp., Tokyo, Japan.
} 

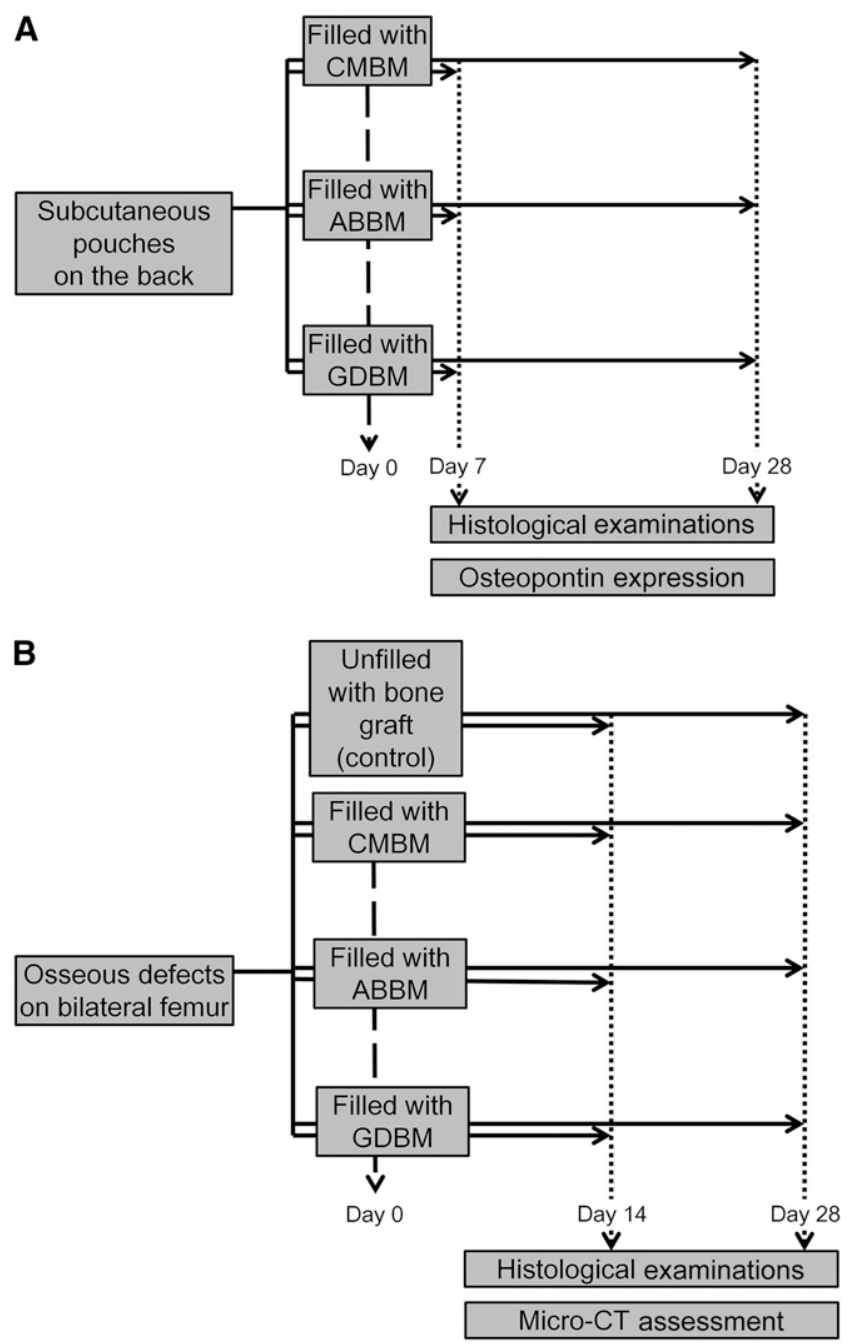

Figure I.

Study design and animal model. Sprague-Dawley rats were used, and six specimens were assessed in each graft material group at each time point. A) Osteoinductivity and biocompatible properties were evaluated by submerging the graft materials into the subcutaneous pouch. B) Osteoconductivity was evaluated by filling the graft materials in the surgically created osseous defect on the femur.

(Tb.N), were separately calculated for each ROI using CT measurement software. ${ }^{\ddagger}$

\section{Histologic Examinations}

After at least 3 days of formaldehyde fixation, all the specimens were decalcified with $12.5 \%$ EDTA for 3 weeks. The specimens were then cut through the midline of the investigated area and embedded in paraffin after dehydration. Three 5 - $\mu \mathrm{m}$ sections were made in the specimens from the subcutaneous pouches. One was stained with hematoxylin and eosin, and the others were stained for osteopontin (OPN), a marker of osteogenesis. One $5-\mu \mathrm{m}$ section was made from femoral specimens and stained with hematoxylin and eosin. All the images were acquired using a digital image acquisition system. $₫ \S$
Quantitative measurements on the specimens from the subcutaneous pouches were performed under $\times 400$ magnification. The thickness of the fibrous capsule was measured in seven randomly selected areas adjacent to the graft materials, and the fractions of inflammatory cells were counted in five randomly selected images adjacent to the graft materials.

Histomorphometric measurements on the femoral specimens were performed using digital measurement software ${ }^{l l l}$ under $\times 100$ magnification (supplementary Fig. 1 in online Journal of Periodontology). The defect fill was defined as the percentage of the wellintegrated osseous tissue, including new bone, marrow space, and grafting materials, to the entire defect. The percentage of each component of the osseous tissue was also calculated. The distribution and intensity of OPN deposition was evaluated in three randomly selected images of each specimen under $\times 400$ magnification. The quantitative measurements were performed by a masked examiner: S-HY for the histomorphometry and Y-HJ for the thickness of fibrous capsule and inflammatory cells. The inflammatory cells were identified based on the cell morphology. All the histologic assessments were supervised by a masked pathologist (Dr. Y-P Wang) from the Pathology Department of the National Taiwan University Hospital.

\section{Statistical Analyses}

One-way analysis of variance followed by Tukey post hoc tests were used to compare the differences of each parameter at each time point. The data are presented as the mean \pm SD of measurements, with a $P$ value $<0.05$ considered statistically significant.

\section{RESULTS}

\section{Histologic Assessments of the Subcutaneous Specimens}

Neither acute inflammation nor tissue necrosis was noted in any of the subcutaneous specimens investigated. At day 7, the graft materials were embedded in fibrous capsule with multinuclear giant cells and inflammatory infiltration in all groups (Figs. 2A through 2C). Histologic artifacts existed at the interface of graft materials and surrounding tissues in the ABBM and CMBM groups owing to the demineralization process (Figs. $2 \mathrm{~B}$ and $2 \mathrm{C}$ ). OPN was prominently expressed in the cells surrounding the graft materials and lightly deposited in the interstitial fibril matrix in the GDBM groups (Fig. 2A). The OPN was similarly distributed but relatively lightly deposited in the CMBM group (Fig. 2C), whereas

‡巿 CTAn, Bruker, Kontich, Belgium.

$\S \S$ AxioCam ICc 5, Carl Zeiss Microscopy, Munich, Germany.

AxioVision, Carl Zeiss Microscopy. 

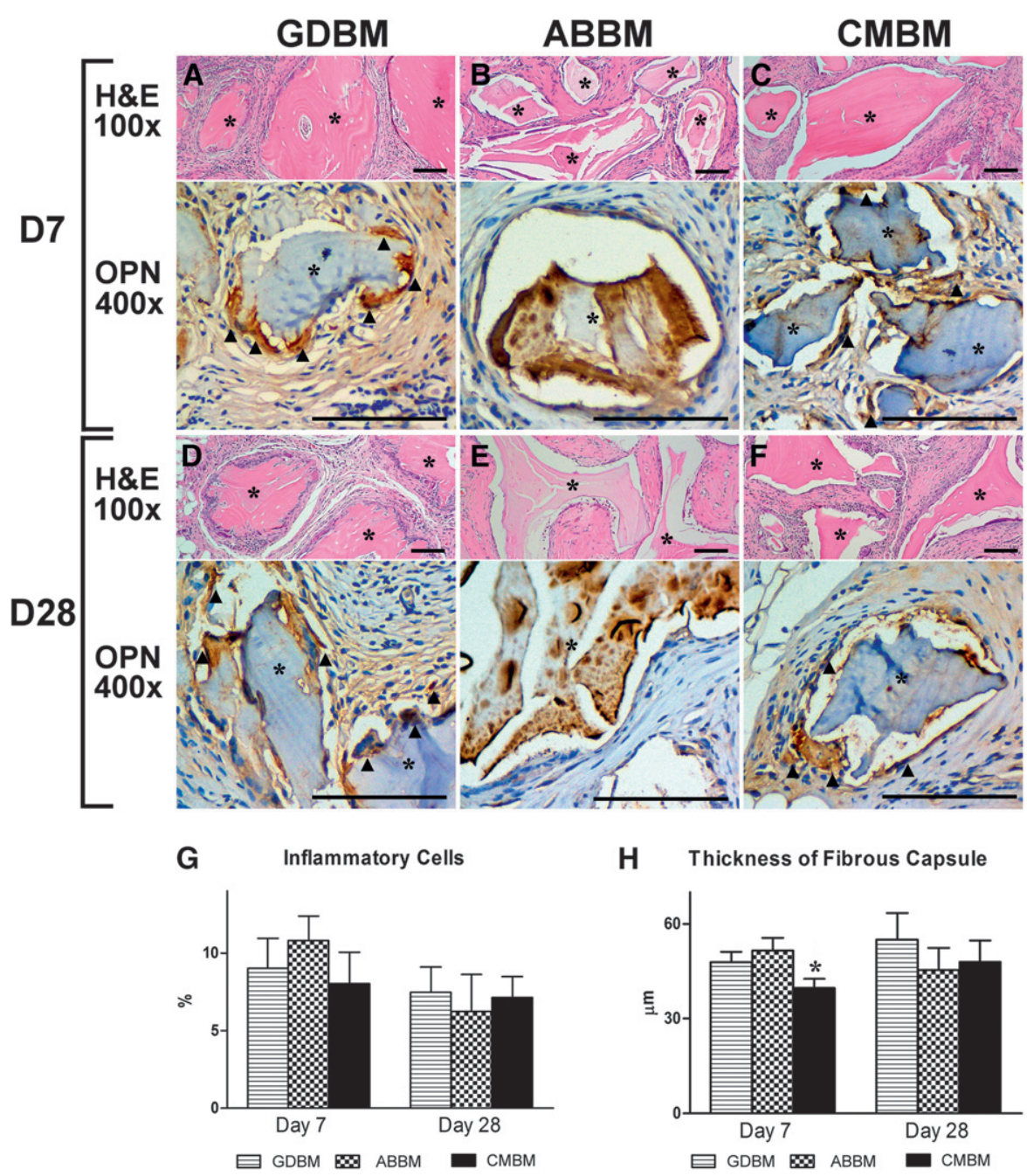

H Thickness of Fibrous Capsule

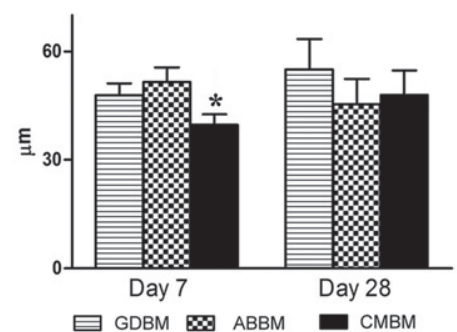

Figure 2.

A through F) Histologic assessments of the subcutaneous specimens at days 7 (D7) and 28 (D28). H\&E = hematoxylin and eosin; scale bar $=100 \mu \mathrm{m}$; asterisk = residual bone grafting materials; arrowheads $=\mathrm{OPN}^{+}$cells. A) Representative specimen from the GDBM group at day 7. B) Representative specimen from the ABBM group at day 7. C) Representative specimen from the CMBM group at day 7. D) Representative specimen from the GDBM group at day 28. E) Representative specimen from the ABBM group at day 28. F) Representative specimen from the CMBM group at day 28. G) Percentage of the inflammatory cells surrounding the grafting materials. H) Averaged thickness of the fibrous capsule surrounding the grafting materials. ${ }^{*} P<0.001$ compared with the GDBM and ABBM groups.

OPN deposition was limited to the residual bone matrix in the ABBM group (Fig. 2B). At day 28, the cellularity as well as inflammatory infiltration was apparently reduced in all groups (Figs. 2D through $2 \mathrm{~F}$ ), whereas multinuclear giant cell deposition on the graft materials was still evident in the GDBM group (Fig. 2D). OPN was still evidently deposited in the cells and fibrils surrounding the graft materials in the GDBM and CMBM groups, and the deposition was still limited to the residual bone matrix in the ABBM group (Figs. 2D through 2F). Therefore, none of the three tested materials showed tissue mineralization at either time point.
The results from the quantitative assessments demonstrated that inflammatory infiltration was minimal in all groups at day 7 and further decreased at day 28 (Fig. 2G). The fibrous capsule surrounding the grafting materials was significantly thinner in the CMBM group than the GDBM and $A B B M$ groups at day 7 , and no significant difference among groups was noted at day 28 (Fig. $2 \mathrm{H})$.

\section{Micro-CT Assessments of the Femoral Specimens}

The border of the osseous defect was distinguishable from the micro$C T$ imaging in all the femoral specimens (Fig. 3A). At day 14, the defect was generally less radiopaque in the control and GDBM groups than the CMBM group, and the ABBM group showed the highest radiopacity (Fig. 3A, upper panel). However, the observations might have just reflected the differences in radiographic density of the grating materials. At day 28, the bone spongiosa was apparently thicker compared with that at day 14 in each group, except the GDBM group (Fig. $3 \mathrm{~A}$, lower panel). The grafting material was still distinguishable from the new bone because of its radiographic density in the $A B B M$ group, whereas the radiographic density of the defect was similar to the native bone in the CMBM group.

Compared with the control, $\mathrm{BV} / \mathrm{TV}$ was significantly lower in the GDBM group $(P<0.05)$ but significantly higher in the ABBM group $(P<0.05)$ at day 14 . At day 28 , only the ABBM group showed significantly higher $\mathrm{BV} / \mathrm{TV}$ relative to the control $(P<0.01$, Fig. $3 \mathrm{~B})$. Significantly higher $\mathrm{Tb}$.Th relative to the control was only noted in the ABBM group at days 14 and 28 $(P<0.01$ and $P<0.001$, Fig. $3 C)$, whereas significantly lower Tb.N relative to the control was only noted in the GDBM group at day $14(P<0.01)$ (Fig. 3D). Therefore, compared with the control, Tb.Sp was significantly higher in the GDBM group at day $14(P<0.01)$ but significantly lower in the ABBM group at day 28 (Fig. 3E). No significant difference 
A

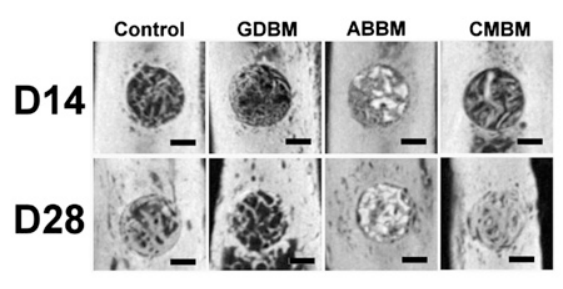

C Trabecular Thickness (Tb.Th)

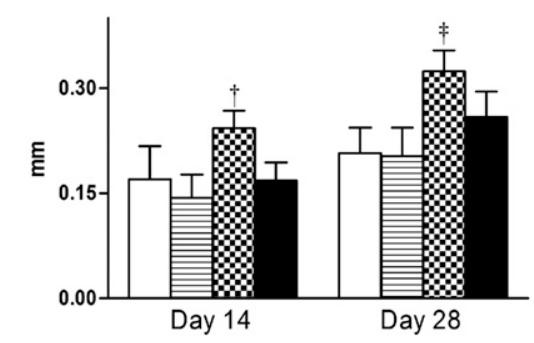

Control $\boxminus$ GDBM $\$$ ABBM $\square$ CMBM
B Relative Bone Volume (BV/TV)

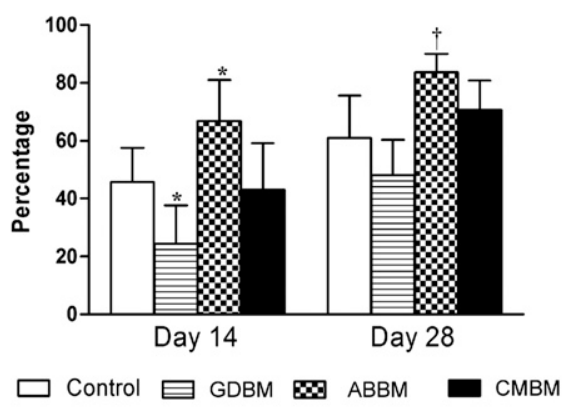

D Trabecular Number (Tb.N)

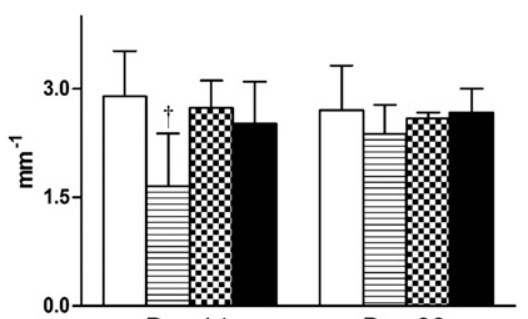

Day 14
Day 28

$\square$ Control $\boxminus$ GDBM $\infty$ ABBM

in the ABBM and CMBM groups. Most grafting materials were bounded by thin lamellated bone and marrow-like structure in the ABBM group (Fig. 4C), whereas the residual graft materials encapsulated by fibrous tissue were evident in three of the six specimens in the CMBM group (Fig. 4D).

At day 28, the defect was nearly completely filled with newly formed bone, and the trabeculae were thicker with smaller lacunar space relative to day 14 in the control group (Fig. 4E). The residual graft materials were still noted in all three other groups, and most of the graft materials were well integrated with the newly formed bone (Figs. 4F through $4 \mathrm{H})$. However, isolated graft materials without bone matrix deposition were still noted in two of the six specimens in the GDBM and CMBM groups (Figs. 4F and $4 \mathrm{H})$.

The defect fill was the highest in the ABBM group at day 14, and complete defect resolution was noted in most specimens at day 28, regardless of the treatment (Fig. 5A). However, no significant differences in defect fill were found among groups at the two time points. The marrow space was slightly higher at day 14 in the control and CMBM groups and apparently decreased at day 28, whereas increasing

was noted in the CMBM group relative to the control in any of the examined micro-CT parameters at either time point.

\section{Histologic Assessments of the Femoral Specimens}

At day 14, the defect was united and partially occupied with newly formed osseous tissue, characterized by thin trabeculae and enlarged osteocytic lacunae, and lined with densely arranged osteoblastic cells in the control group (Fig. 4A). The newly formed bone was well integrated to the residues of the demineralized bone matrix, and the formation of a marrow-like structure was noted in the GDBM group (Fig. 4B). The histologic artifact caused by the demineralization process was noted marrow space was noted in the GDBM group at day 28 (Fig. 5B). However, no significant differences in percentage of marrow space area were found among groups at either time point. The percentage of bone component in the CMBM group was significantly lower at day 14 than the control group. The difference was not statistically significant at day 28 . The percentage of bone component of the GDBM group was statistically significantly lower than the control group at day 28 (Fig. 5C).

\section{DISCUSSION}

In the first part of the study, minimal inflammation and well-defined fibrous encapsulation were observed in all three groups at both time points (Figs. $2 \mathrm{G}$ and $2 \mathrm{H}$ ), indicating that all the examined graft 
materials presented acceptable biocompatibility. OPN, a protein regulating the formation and remodeling of mineralized tissue, ${ }^{23}$ was highly expressed in the early stage in the GDBM group, implying that signaling for osteogenesis was initialized. However, no active mineralization was noted in any specimen investigated, including the GDBM group. Although growth factors have been known to enhance the osteogenic process, and BMPs have been shown to be fundamentally important regulators of osteogenesis and osseous defect repair, ${ }^{24}$ the concentration of the growth factor in the GDBM $(<30 \mathrm{ng} / \mathrm{g}$ BMP-2 and $<0.7 \mathrm{ng} / \mathrm{g}$ BMP-4) might not be sufficient to trigger the process of mineralization, and at least 600 ng BMP-2 and 100 ng BMP-4 may be needed in vivo. ${ }^{24,25}$ The fact that the bone grafting materials were not exactly confined in the subcutaneous tissue and that the local concentration of growth factors might be diluted and further reduces their osteoinductive potential.

In the second part of the study, the micro-CT assessments showed higher BV/TV and Tb.Th in the ABBM group (Figs. 3B and $3 C$ ), and lower Tb.N and higher Tb.Sp was associated with the GDBM group compared with the ABBM and CMBM groups (Fig. 3). Although the micro-CT imaging provided three-dimensional structural configuration and showed results comparable to the conventional histology, ${ }^{26}$ all the analyses relied on only radiodensity and might need special attention when interpreting results in the presence of bone grafting materials. For instance, ABBM and CMBM are radiopaque materials and are more likely to lead to overestimation of the quantity of newly formed bone, and the performance of the GDBM group might be underestimated since it is more radiolucent. Thus, although lower BV/TV and Tb.N values were noted in the GDBM group relative to the control group at day 14 (Figs. 3B and 3D), it could not be inferred that the osteogenic property of the GDBM
A

Defect Fill

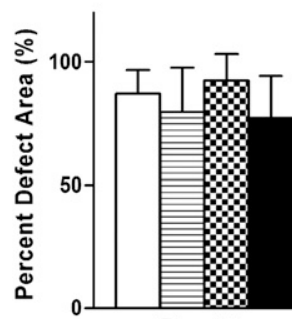

Day 14

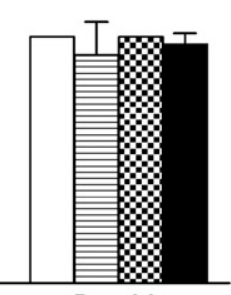

Day 28

$\square$ Control $\boxminus$ GDBM $\otimes$ ABBM

B

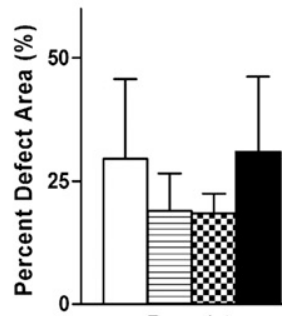

Day 14

$\square$ Control $\boxminus$ GDBM $\infty$ ABBM

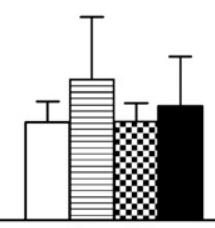

Day 28

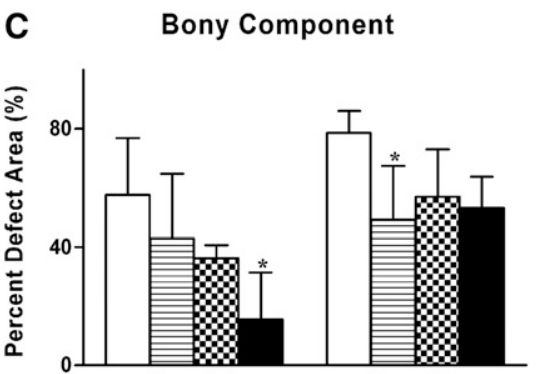

Day 14

Day 28

Figure 5.

Histomorphometry of the femoral specimens. A) Percentage of defect fill. B) Percentage of the marrow space of the osseous defect. C) Percentage of the defect area solely occupied with the bony structure. *P $<0.05$ compared with the control group. 
group was inferior. In this regard, supplemental histologic evidence is necessary. All examined groups demonstrated similar levels of histologic defect fill (Fig. 5A), and because of the presence of the residual grafting matrix, the real bone compartment in all the grafted groups (i.e., GDBM, ABBM, and CMBM) was lower relative to the control group (Fig. $5 C)$. By subtracting residual grafting materials, all grafting groups (i.e., GDBM, ABBM, and CMBM) showed statistically comparable levels of marrow space area as well as the area of bony component (Figs. 5B and 5C), and the newly formed bone was well integrated with grafting materials at day 28 (Fig. 4). The results support that ABBM and CMBM have similar osteoconductive potential, which had been reported in previous investigations, ${ }^{27,28}$ and indicate that the osteoconductivity of GDBM was equivalent with that of ABBM and CMBM. Although it is generally accepted that $A B B M$ could exhibit osteogenic potential equivalent to DFDBA, ${ }^{29}$ in this study, GDBM demonstrated equivalent osteoconductivity relative to ABBM (Figs. 5A through $5 \mathrm{C}$ ), implying that the effect of growth factor was not significant, and it could be associated with the fast degradation or dilution of the growth factors. Specifically, rapid release of growth factors within the first 2 days from the bone replacement grafts was usually observed in several in vitro investigations. ${ }^{30,31}$ Furthermore, osteogenesis involves a cascade of physiologic events timely mediated by distinct growth factors. To maximize the osteogenic capability of each growth factor, developing a biocompatible scaffold to control the release of growth factors in appropriate stages is needed. For instance, by using a bilayered microsphere composed of poly-(D,L-lactide) and poly-(D,L-lactide-coglycolide) to encapsulate an early (e.g., PDGF) and a late (e.g., simvastatin) bioactive molecule in the respective compartment, the osteogenesis as well as periodontal regeneration could be accelerated by the controlled sequential release of bioactive molecules. ${ }^{32}$

Limitations of this study are: 1) traditional DFDBA was not available for comparison; 2) critical-sized defects were unable to be made in femurs due to the small size of the femurs; and 3) false-positive results are possible from statistical analyses. In addition, these surgically created acute defects in animals with high healing capability might not be representative of human dentoalveolar defects.

\section{CONCLUSIONS}

Notwithstanding the limitations of the study, GDBM demonstrated slightly greater osteoinductivity according to the relatively prominent deposition of OPN, and its osteoconductivity as well as biocompatibility was equivalent to those of ABBM. From a clinical point of view, the selection of the abovementioned materials should be based on other factors, such as cost, ease of manipulation, and clinician preference. Further investigations are warranted to confirm the additive effect of growth factors in the DFDBA and develop a suitable scaffold to control the release of growth factors in a more clinically relevant model.

\section{ACKNOWLEDGMENTS}

The authors acknowledge the technical support from the Pathology Department of National Taiwan University Hospital. The study was supported by a research grant (101R7465) from National Taiwan University. The authors report no conflicts of interest related to this study.

\section{REFERENCES}

1. Schwartz Z, Doukarsky-Marx T, Nasatzky E, et al. Differential effects of bone graft substitutes on regeneration of bone marrow. Clin Oral Implants Res 2008; 19:1233-1245.

2. Fuentes R, Venegas GO, Olate S, Rauco MR, Borie E, Engelke W. Histological evaluation of bone regeneration means freeze dried bone allograft (FDBA) in post exodontia sockets. Int J Morphol 2011;29:286-292.

3. Brunsvold MA, Mellonig JT. Bone grafts and periodontal regeneration. Periodontol 2000 1993;1:80-91.

4. Bauer TW, Muschler GF. Bone graft materials. An overview of the basic science. Clin Orthop Relat Res 2000;(371):10-27.

5. Myeroff C, Archdeacon M. Autogenous bone graft: Donor sites and techniques. J Bone Joint Surg Am 2011;93:2227-2236.

6. Younger EM, Chapman MW. Morbidity at bone graft donor sites. J Orthop Trauma 1989;3:192-195.

7. Sanders JJ, Sepe WW, Bowers GM, et al. Clinical evaluation of freeze-dried bone allografts in periodontal osseous defects. Part III. Composite freeze-dried bone allografts with and without autogenous bone grafts. J Periodontol 1983;54:1-8.

8. Rummelhart JM, Mellonig JT, Gray JL, Towle HJ. A comparison of freeze-dried bone allograft and demineralized freeze-dried bone allograft in human periodontal osseous defects. J Periodontol 1989;60: 655-663.

9. Schwartz Z, Somers A, Mellonig JT, et al. Ability of commercial demineralized freeze-dried bone allograft to induce new bone formation is dependent on donor age but not gender. J Periodontol 1998;69:470-478.

10. Richardson CR, Mellonig JT, Brunsvold MA, McDonnell HT, Cochran DL. Clinical evaluation of Bio-Oss: A bovine-derived xenograft for the treatment of periodontal osseous defects in humans. J Clin Periodontol 1999;26:421-428.

11. Lieberman JR, Daluiski A, Einhorn TA. The role of growth factors in the repair of bone. Biology and clinical applications. J Bone Joint Surg Am 2002;84-A:10321044.

12. Selvig KA, Sorensen RG, Wozney JM, Wikesjö UM. Bone repair following recombinant human bone morphogenetic protein-2 stimulated periodontal regeneration. J Periodontol 2002;73:1020-1029. 
13. Nevins $M$, Camelo $M$, Nevins ML, Schenk RK, Lynch SE. Periodontal regeneration in humans using recombinant human platelet-derived growth factor-BB (rhPDGF-BB) and allogenic bone. J Periodontol 2003; 74:1282-1292.

14. Wikesjö UM, Qahash M, Polimeni G, et al. Alveolar ridge augmentation using implants coated with recombinant human bone morphogenetic protein-2: Histologic observations. J Clin Periodontol 2008;35:1001-1010.

15. Andrew JG, Hoyland JA, Freemont AJ, Marsh DR. Platelet-derived growth factor expression in normally healing human fractures. Bone 1995;16:455-460.

16. Dereka XE, Markopoulou CE, Mamalis A, Vrotsos IA. Effect of rhBMP-7 combined with two bone grafts on human periodontal ligament cell differentiation. Growth Factors 2009;27:274-279.

17. Bowers G, Felton F, Middleton C, et al. Histologic comparison of regeneration in human intrabony defects when osteogenin is combined with demineralized freeze-dried bone allograft and with purified bovine collagen. J Periodontol 1991;62:690-702.

18. Boyan BD, Ranly DM, Schwartz Z. Use of growth factors to modify osteoinductivity of demineralized bone allografts: Lessons for tissue engineering of bone. Dent Clin North Am 2006;50:217-228, viii.

19. Chang PC, Chong LY, Dovban AS, et al. Sequential platelet-derived growth factor-simvastatin release promotes dentoalveolar regeneration. Tissue Eng Part A 2014;20:356-364.

20. Chang PC, Chung MC, Lei C, Chong LY, Wang $\mathrm{CH}$. Biocompatibility of PDGF-simvastatin double-walled PLGA (PDLLA) microspheres for dentoalveolar regeneration: A preliminary study. J Biomed Mater Res A 2012;100:2970-2978.

21. Burghardt AJ, Kazakia GJ, Laib A, Majumdar S. Quantitative assessment of bone tissue mineralization with polychromatic micro-computed tomography. Calcif Tissue Int 2008;83:129-138.

22. Chang PC, Liang K, Lim JC, Chung MC, Chien LY. A comparison of the thresholding strategies of micro-CT for periodontal bone loss: A pilot study. Dentomaxillofac Radiol 2013;42:66925194.

23. Denhardt DT, Guo X. Osteopontin: A protein with diverse functions. FASEB J 1993;7:1475-1482.

24. Wozney JM, Rosen V. Bone morphogenetic protein and bone morphogenetic protein gene family in bone formation and repair. Clin Orthop Relat Res 1998; (346):26-37.

25. Peng H, Wright V, Usas A, et al. Synergistic enhancement of bone formation and healing by stem cellexpressed VEGF and bone morphogenetic protein-4. $J$ Clin Invest 2002;110:751-759.

26. Park CH, Abramson ZR, Taba M Jr., et al. Threedimensional micro-computed tomographic imaging of alveolar bone in experimental bone loss or repair. J Periodontol 2007;78:273-281.

27. Schmitt CM, Doering H, Schmidt T, Lutz R, Neukam FW, Schlegel KA. Histological results after maxillary sinus augmentation with Straumann ${ }^{\circledR}$ BoneCeramic, Bio-Oss ${ }^{\circledR}$, Puros ${ }^{\circledR}$, and autologous bone. A randomized controlled clinical trial. Clin Oral Implants Res 2013;24:576-585.

28. Froum SJ, Wallace SS, Elian N, Cho SC, Tarnow DP. Comparison of mineralized cancellous bone allograft (Puros) and anorganic bovine bone matrix (Bio-Oss) for sinus augmentation: Histomorphometry at 26 to 32 weeks after grafting. Int J Periodontics Restorative Dent 2006;26:543-551.

29. Jensen SS, Terheyden H. Bone augmentation procedures in localized defects in the alveolar ridge: Clinical results with different bone grafts and bonesubstitute materials. Int $J$ Oral Maxillofac Implants 2009;24(Suppl.):218-236.

30. Pietrzak WS, Dow M, Gomez J, Soulvie M, Tsiagalis G. The in vitro elution of BMP-7 from demineralized bone matrix. Cell Tissue Bank 2012;13:653-661.

31. Gille J, Dorn B, Kekow J, Bruns J, Behrens P. Bone substitutes as carriers for transforming growth factor-beta(1) (TGF-beta(1)). Int Orthop 2002;26: 203-206.

32. Chang P-C, Dovban AS, Lim LP, Chong LY, Kuo MY, Wang C-H. Dual delivery of PDGF and simvastatin to accelerate periodontal regeneration in vivo. Biomaterials 2013;34:9990-9997.

Correspondence: Dr. Po-Chun Chang, Graduate Institute of Clinical Dentistry, School of Dentistry, National Taiwan University, 1 Chang-Te St, Taipei 100, Taiwan. Fax: +8862-2383-1346; e-mail: changpc@ntu.edu.tw.

Submitted June 4, 2014; accepted for publication July 21, 2014. 